\title{
Aprovechamiento múltiple de los frutos de EUterPe EDULIS MART. (ARECACEAE): LA CONSERVACIÓN DEL PODER GERMINATIVO COMO ASPECTO CLAVE EN EL MANEJO DE LA ESPECIE
}

\author{
Euterpe edulis MART. (ARECACEAE) Fruits MULtiple uses: The \\ CONSERVATION OF GERMINATIVE POWER AS A KEY ASPECT FOR ITS MANAGEMENT
}

\author{
Sofia Lindner ${ }^{1}\left[\right.$, Daily S. García ${ }^{2,3}$, Peggy Thalmayr ${ }^{2 *}\left(\left[\right.\right.$ y Norma I. Hilgert ${ }^{2,3}$
}

1. Lic. en Gestión Ambiental. Consultora independiente con énfasis en extensión rural y educación ambiental, Puerto Iguazú, Misiones, Argentina. 2. Instituto de Biología Subtropical, Universidad Nacional de Misiones, CONICET. Asoc. Centro de Investigaciones del Bosque Atlántico (CelBA). Bertoni 85, Puerto Iguazú, Misiones, Argentina.

3. Facultad de Ciencias Forestales, Universidad Nacional de Misiones. Bertoni 124. Eldorado, Misiones, Argentina.

\section{*peggythalmayr@gmail.com}

Citar este artículo LINDNER, S., D. S. GARCÍA, P. THALMAYR \& N. I. HILGERT. 2021. Aprovechamiento múltiple de los frutos de Euterpe edulis Mart. (Arecaceae): La conservación del poder germinativo como aspecto clave en el manejo de la especie. Bol. Soc. Argent. Bot. 56: 467-478.

DOI: https://doi. org/10.31055/1851.2372.v56. n4.32130

Recibido: 10 Feb 2021

Aceptado: 5 Set 2021

Publicado en línea: 15 Nov 2021

Publicado impreso: 20 Dic 2021

Editor: Federico G. O. Mollard (D)

ISSN versión impresa 0373-580X

ISSN versión on-line 1851-2372

\section{SUMMARY}

Background and aims: Euterpe edulis is an endemic palm tree of the Atlantic Forest whose seeds germinative power decreases when dehydrated because they are recalcitrant. In the past, the plants were cut -which implied death- to harvest the foliar sprout for food purposes, nowadays, fruits are marketed as food and seeds for the ornamental market. To evaluate whether this double use of fruits is possible with local management practices, tests were carried out on the germination power and the survival of seedlings.

M\&M: We evaluated germination percentage, seed germination speed index, and seedling survival percentage in fruits either stored with pulp or pulped. In addition, the relative increase in the number of pulped fruits was valued by $1 \mathrm{~kg}$, to estimate the commercial value of this new presentation.

Results: No significant differences were found in the germination power and survival of seedlings from fruits stored whole or pulped. However, the seeds of pulped stored fruits germinated faster. An increase of $33 \%$ of pulped fruits in $1 \mathrm{~kg}$ was estimated.

Conclusions: The storage of whole or pulped fruits does not affect germination of $E$. edulis seeds, nor the survival of the seedlings, so double use of the fruits is possible. It is recommended to increase the value by $50 \%$ per $\mathrm{kg}$ of pulped fruits, concerning the commercial value of the whole fruits.

\section{KEY WORDS}

Atlantic Forest, edible fruit, jejy'a, local management, Palmito, productive diversification.

\section{RESUMEN}

Introducción y objetivos: Euterpe edulis es una palmera endémica del Bosque Atlántico cuyo poder germinativo de las semillas disminuye ante la deshidratación por ser recalcitrantes. En el pasado, los ejemplares se cortaban -lo que implicaba la muerte- para cosechar el brote foliar con destino alimenticio, en el presente, se comercializan la pulpa de los frutos como alimento y las semillas para el mercado ornamental. Para evaluar si este doble aprovechamiento de los frutos es posible con las prácticas locales de manejo, se realizaron ensayos sobre el poder germinativo y la supervivencia de plantines.

M\&M: En frutos almacenados con pulpa y despulpados, se comparó el porcentaje de germinación, el índice de velocidad de germinación en semillas y el porcentaje de supervivencia de plántulas. Además, se calculó el aumento relativo del número de frutos despulpados en $1 \mathrm{~kg}$, para estimar el valor comercial de esta nueva presentación.

Resultados: No se hallaron diferencias significativas en el poder germinativo y supervivencia de plántulas provenientes de frutos almacenados enteros o despulpados. Sin embargo, las semillas de frutos almacenados despulpados germinaron con mayor velocidad. Se estimó un aumento de un $33 \%$ de frutos despulpados en $1 \mathrm{~kg}$.

Conclusiones: El almacenamiento de los frutos enteros o despulpados no afecta a la germinación de las semillas de E. edulis, ni la sobrevida de las plántulas, por lo que es posible el doble aprovechamiento de los frutos. Se recomienda incrementar en un $50 \%$ el valor del $\mathrm{kg}$ de frutos despulpados, respecto al valor comercial de los frutos enteros.

\section{Palabras clave}

Bosque Atlántico, diversificación productiva, jejy’a, manejo local, Palmito, pulpa comestible. 


\section{INTRODUCCIÓN}

Las palmeras del género Euterpe representan recursos económicos y culturales claves para la subsistencia de los pueblos y la fauna que habitan en su área de distribución; dentro de los usos humanos más generalizados se destacan el alimenticio, ornamental, medicinal y para la construcción (Reis et al., 2000; Clement et al., 2005; Keller, 2009; Barroso et al., 2010; Milanesi et al., 2013; García et al., 2020). Euterpe edulis Martius es la especie del género con distribución más austral y endémica del Bosque Atlántico (en Paraguay, Brasil y Argentina) (Henderson, 2000). Este bioma que ha sido clasificado como una de las zonas calientes de biodiversidad del mundo y como uno de los ecosistemas más amenazados dado el alto grado de fragmentación de sus remanentes (Myers et al., 2000).

El aprovechamiento tradicional más difundido de E. edulis (conocido en Argentina como palmito y en Brasil como jussara), consiste en el corte del extremo apical de la planta, la extracción del cogollo y su consumo como alimento; manipulación que implica la muerte del individuo (ya que allí se aloja la yema apical y por lo tanto la planta ya no rebrota) y un consecuente empobrecimiento y disminución de las poblaciones silvestres manejadas (Reis et al. 2000; Chediack, 2008). En ese contexto, fue cobrando importancia la cosecha de los frutos, puesto que su colecta no implica el corte de las palmeras. Así, en líneas generales, en Brasil se emplea la pulpa de los frutos (ectocarpo y mesocarpo) como alimento (Trevisan et al., 2015) y en la provincia de Misiones, en Argentina, las semillas, con el objeto de producir plantines con fines ornamentales (Furlan et al., 2015; García et al., 2020). Según la literatura, en Brasil el consumo de la pulpa se inspiró en el açaí, el que es extraído de Euterpe oleracea; en el presente, se reconoce que los frutos de ambas especies aportan elementos beneficiosos para la salud, especialmente en enfermedades coronarias, anemia y sobrepeso (De Brito et al., 2007; Kang et al., 2010; Rufino et al., 2010; Poulose et al., 2012; Felzenszwalb et al., 2013; Schulz et al., 2016; Silva et al., 2021).

El manejo de los frutos con fines reproductivos implica ciertos retos. Por un lado, la naturaleza recalcitrante de las semillas pone en riesgo el mantenimiento de la viabilidad reproductiva ante el almacenamiento prolongado (Reis et al., 1999;
Queiroz, 2000; Martins et al., 2004). En este aspecto, según los estudios de Reis et al. (1999) y Cursi \& Cicero (2014), la deshidratación es la principal causa de la pérdida de viabilidad y muerte de las semillas, pues tenores de humedad debajo del $28 \%$ afectan significativamente su germinación. Por otra parte, esta especie tiene un tiempo de germinación lento y heterogéneo. Característica atribuible a la barrera hidrófuga que implica la pulpa oleaginosa y con ello la lenta penetración del agua en el interior del fruto ya que dicha pulpa es un sustrato rico para el desarrollo de microorganismos que pueden afectar al embrión y, de ese modo, modificar el porcentaje final de emergencia (Bovi \& Cardoso, 1976; Bovi, 1990).

Según estudios realizados en Brasil, para acelerar y sincronizar la germinación se recomienda despulpar a los frutos, tratándose de un método accesible y de bajo costo (Bovi \& Cardoso, 1976; Bovi, 1990; Aguiar et al., 2017; Guimarães et al., 2018). En numerosos ensayos se registró que los frutos despulpados y en determinadas condiciones de almacenamiento, conservan el poder germinativo e incluso presentan mayor porcentaje de germinación en relación a frutos almacenados enteros. Entre ellos, Queiroz (2000) explica que los frutos necesitan ambientes adecuados para su almacenamiento, como contenedores especiales y que el período no se extienda por más de cinco meses para asegurar la viabilidad de las semillas. Por otra parte, Bovi (1990) demostró que frutos mantenidos a la sombra en bolsas plásticas por tres días y luego despulpados presentan un porcentaje de emergencia mayor (72 a 97\%) que frutos no despulpados (44 a 69\%). También, Cursi \& Cicero (2014) no observaron diferencias significativas en el desempeño fisiológico de las semillas entre el despulpado manual y el mecánico.

En la provincia de Misiones, Argentina, las familias de la colonia Andresito, dentro de una economía diversificada, desde hace una década aproximadamente comercializan los frutos del palmito con destino a la producción de plantines con fines ornamentales, modelo productivo gestado enteramente por los productores y ya consolidado en el tiempo. Desde el año 2018, se ha comenzado a vender también la pulpa extraída de los frutos (denominada localmente jejy'a). La combinación de estos dos productos obtenidos de los mismos frutos, representa una alternativa de mejora en los 


\section{S. Lindner et al. - Aprovechamiento múltiple de E. edulis y conservación del poder germinativo}

ingresos familiares y, a la vez un modo de promover la conservación de la especie y el bosque cuyo dosel favorece su reclutamiento. Además, representa una oportunidad para empoderar a las mujeres de la colonia Andresito y reforzar la diversificación sostenible de las producciones locales (Hilgert et al., 2019; García et al., 2020).

Frente a la posibilidad de extraer la pulpa de los mismos frutos que se destinan a fines reproductivos, se requirieron ensayos que confirmen que, en el contexto ambiental y de manejo local, los frutos despulpados también mantengan las semillas viables. En este trabajo se presentan los resultados de dichos ensayos realizados con el objetivo de evaluar el efecto en el poder germinativo de las semillas, del almacenamiento según las prácticas de manejo locales de los frutos con y sin pulpa de E. edulis. Nos referimos como manejo local al proceso de cosecha y posterior acopiado de los frutos en bolsas en el sotobosque a la espera de los compradores, entre 20 días a un mes. A su vez, puesto que en un volumen/ peso definido de frutos con pulpa se pueden alojar más frutos sin pulpa (es decir más semillas), se estimó el aumento relativo del número de frutos contenidos en un volumen/peso de frutas sin pulpa, de modo de sugerir el ajuste requerido al costo de las unidades de venta de frutos despulpados.

\section{Materiales y Métodos}

Se realizaron ensayos de germinación, para analizar como varía el poder germinativo de semillas de Euterpe edulis provenientes de frutos que fueron almacenados con pulpa (tal como es el manejo actual) y sin pulpa (despulpados mecánicamente). Para ello se adquirieron dos bolsas de $50 \mathrm{~kg}$ de frutos de productores de Península de Andresito, Misiones (2531'38.26”'S; 547'28.80'O, con alturas que van desde los 200 msnm, en las márgenes del Río Iguazú, hasta los $240 \mathrm{msnm}$ en su porción sur) cosechados en mayo de 2017. El mismo día fueron trasladados hacia la Reserva Karadya, Misiones (2551'34.33”S; $53^{\circ} 57^{\prime} 38.80^{\prime \prime}$ ) donde se realizó el ensayo.

El primer paso consistió en mezclar los frutos en un solo conjunto y luego dividirlos en ocho bolsas con igual peso. Los frutos de cuatro bolsas se almacenaron enteros y los de las bolsas restantes fueron despulpados de forma mecánica antes del almacenamiento previo. Las ocho bolsas se almacenaron debajo de árboles, dentro del bosque, en condiciones similares y cuidando que las bolsas de ambos tratamientos estén dispuestas entremezcladas. Este tipo de almacenamiento emula el modo local de acopio.

\section{Despulpado mecánico}

Se empleó una despulpadora de frutas con paletas, marca Metvisa (metalúrgica Metvisa, Brusque, SC, Brasil), con capacidad de 20 litros. Todos los frutos de este tratamiento fueron despulpados al inicio del ensayo. Para el despulpado mecánico los frutos sanos, maduros y completos fueron seleccionados, lavados con agua corriente, luego fueron sumergidos en agua limpia y mantenidos allí durante 12 horas. Posteriormente se volvieron a lavar agregando 5 $\mathrm{ml}$ de lavandina comercial $(20 \mathrm{~g} \mathrm{Cl} / \mathrm{l})$ por litro de agua. Para el despulpado mecánico se efectuaron los siguientes pasos: 1) Se limpiaron y esterilizaron con alcohol medicinal los utensilios, 2) Se enjuagaron y pesaron los frutos para determinar la cantidad de agua potable que se necesitaría para el proceso, 3) Se colocaron en cada etapa $8 \mathrm{~kg}$ de frutos dentro de la máquina despulpadora y 41 de agua, 4) Se encendió la máquina y se arrojó el agua de a poco. El tiempo de despulpado se controló con un temporizador. En promedio fueron 3,5 minutos, 5) Se apagó la máquina, 6) Se envasó la pulpa en bolsas de plástico, 7) Se volvió a encender la máquina para retirar las semillas y restos de pulpa y 8) Toda la pulpa se guardó en un congelador. En cada ronda de despulpado se tomaron diez semillas al azar para observar a simple vista si en el proceso se les había ocasionado algún daño.

\section{Despulpado manual}

Los frutos almacenados con pulpa fueron despulpados de modo manual antes de cada siembra. Para ello fueron sumergidos 12 horas en un balde con agua a menos de $40^{\circ} \mathrm{C}$ (previamente hervida), luego fueron restregados suavemente entre sí con las manos. Las semillas se enjuagaron con abundante agua una vez que la superficie de las mismas se observaba sin pulpa.

\section{Ensayo de germinación}

Con las semillas almacenadas de las dos formas antes explicadas se realizaron cuatro siembras con diez días de diferencia entre cada una. Las semillas provenientes de los frutos almacenados con y $\sin$ 
pulpa se desinfectaron antes de ser sembradas, para ello fueron sumergidas durante 10 minutos en solución acuosa al 10\% de hipoclorito de sodio (para evitar el desarrollo de hongos) y durante 15 minutos en agua a $40^{\circ} \mathrm{C}$ (para homogeneizar y acelerar el proceso de germinación).

La primera siembra (0) se efectuó al día siguiente de la cosecha, para ello se seleccionaron 100 frutos de cada condición de almacenamiento. Luego se repitieron siembras a los 10, 20 y 30 días (siembras 1,2 y 3 respectivamente) de almacenamiento de los frutos en el monte; nuevamente, se tomaron en cada caso 100 frutos de cada tratamiento. En todos los casos la siembra se realizó en bandejas plásticas, con diez semillas cada una, y por lo tanto diez bandejas por tratamiento. El sustrato utilizado fue corteza de pino compostada y perlita, el que previo a la siembra fue secado por 15 minutos en el microondas y humedecido con agua. Una vez depositadas las semillas, las bandejas fueron regadas y cubiertas con una bolsa plástica para mantener la humedad. Finalmente fueron colocadas en estantes bajo techo, donde recibieron luz natural a lo largo del día. No se controló la temperatura, ni la humedad ambiente del lugar del ensayo.

Cuando el desarrollo de la radícula era evidente (estadio 3, ver más abajo), cada semilla fue trasplantada y ubicada en un vivero rústico situado en un sitio sombreado al aire libre. Las plántulas fueron colocadas en una bolsa de plástico de 25 x 30 $\mathrm{cm}$ con sustrato de tierra tamizada y restos de troncos en descomposición. Cada plántula fue identificada y etiquetada de modo de mantener la trazabilidad.

\section{Toma y análisis de datos}

Se hizo un seguimiento semanal del desarrollo de las plántulas durante 20 semanas, iniciando a los 7 días de la primera siembra (0) -26 de mayo-. Se tuvieron en cuenta ocho estadios de desarrollo (Fig. 1) para las evaluaciones, siguiendo la metodología empleada por Queiroz (2000) y descripciones de Tomlinson (1990):

Estadio 1. Imbibición de las semillas. (Fig. 1A).

Estadio 2. Primera manifestación externa de la germinación. Desplazamiento de la película endocárpica que cubre al embrión, seguido por la emergencia del botón germinativo constituido por gémula y radícula envueltos por tejidos cotiledonares. (Fig. 1B).
Estadio 3. Emergencia de la primeraraíz(embrionaria) que se desarrolla perpendicularmente al eje caulinar. Rápidamente sufre torción por geotropismo positivo. (Fig. 1C).

Estadio 4. Emergencia de la primera hoja restringida a una vaina rígida de coloración rosada, provocando la emergencia de la gémula por una grieta cotiledonar. La plántula que surge se encuentra adherida a la semilla (germinación admotiva). (Fig. 1D).

Estadio 5. Emergencia y elongación de raíces adventicias. La primera raíz puede sufrir el aborto y ser sustituida por una nueva raíz adventicia más robusta. (Fig. 1E).

Estadio 6. Emergencia de la segunda hoja, casi totalmente reducida a una vaina y con el limbo pequeño. (Fig. 1F).

Estadio 7. Plántulas con la tercera hoja emergente (primera hoja fotosintética). (Fig. 1G).

Estadio 8. Plántula con el limbo foliar desplegado. (Fig. 1H).

Las semillas fueron dadas por muertas cuando comenzaban a descomponerse, estaban vacías o el embrión se observaba oscuro, tomando en cuenta a Reis (1995), quien sostiene que cualquiera de estos procesos representa su inviabilidad y son rasgos indicadores efectivos.

Una vez finalizadas las observaciones, se estimó el porcentaje de germinación de las semillas $(\% \mathrm{G})$, es decir el número de semillas germinadas $(\mathrm{N})$ evaluado semanalmente, en relación al número total de semillas sembradas (A), expresado en porcentaje (Gómez Restrepo, 2004). Además, se calculó el índice de velocidad de germinación (IVG) según la ecuación de Maguire (1962):

$$
I V G=\sum_{i=1}^{n} \frac{x_{i}}{N_{i}}
$$

En donde, xi representa el número de semillas germinadas por día, $\mathrm{Ni}$ el número de días después de la siembra y $\mathrm{n}$ el número de conteos $1,2 \ldots, \mathrm{n}$ conteos.

Por otra parte, se estimó el porcentaje de supervivencia de las semillas $(\% \mathrm{~S})$, es decir el número de semillas viables en relación al total de semillas sembradas (A) expresado en porcentaje. Se consideran como semillas viables aquellas -germinadas o no- que no estuvieran vacías, con el embrión oscuro o descompuestas. Observando a simple vista y palpando con los dedos se puede 

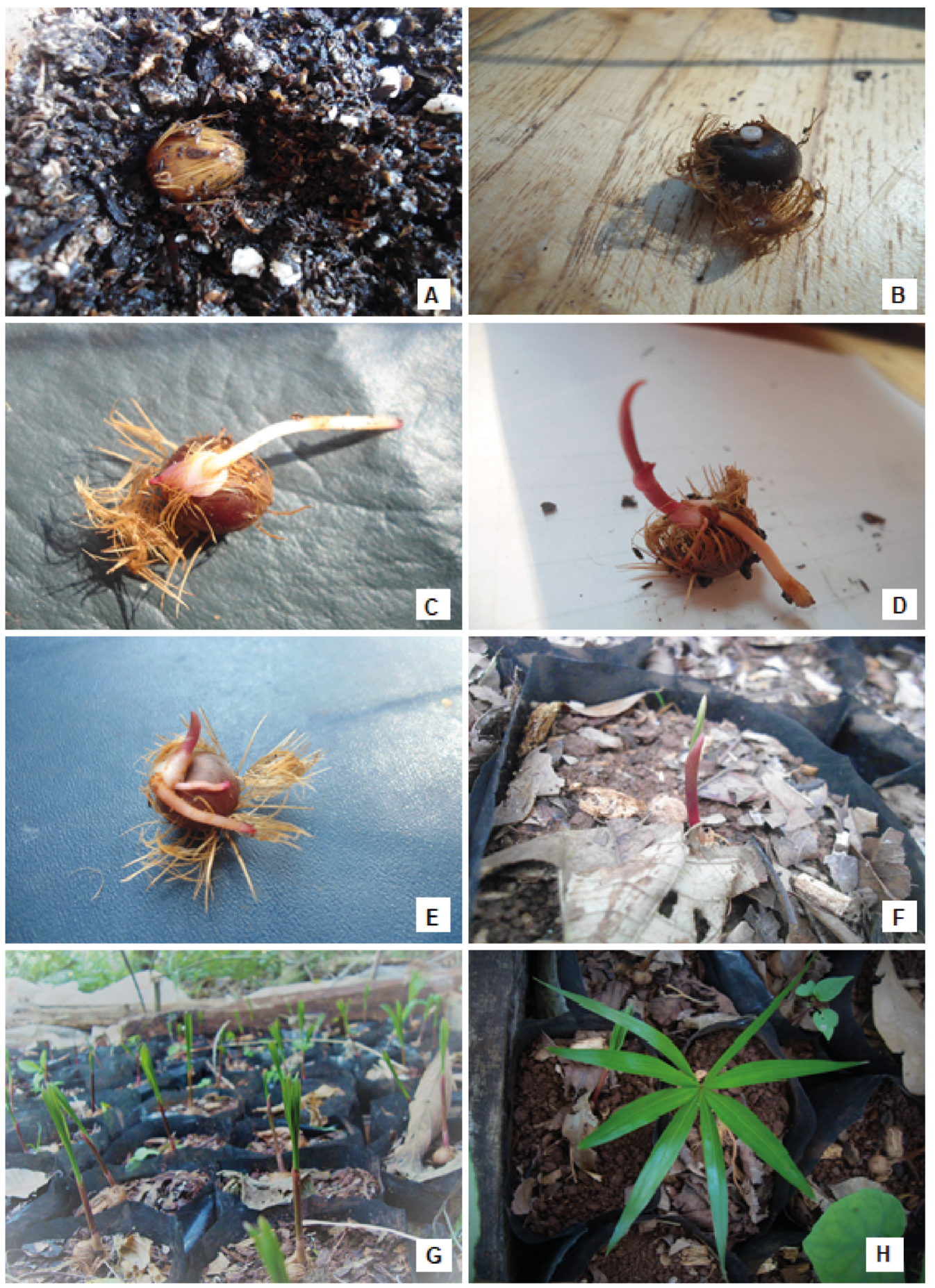

Fig. 1. Estadios de desarrollo durante la germinación y emergencia de E. edulis considerados durante el seguimiento del ensayo. A: Estadio 1. Imbibición de las semillas B: Estadio 2. Emergencia del botón germinativo. C: Estadio 3. Emergencia de la raíz embrionaria. D: Estadio 4. Emergencia de la primera hoja. E: Estadio 5. Emergencia de raíces adventicias F: Estadio 6. Emergencia de la segunda hoja. G: Estadio 7. Emergencia de la tercera hoja H: Estadio 8. Plántula con el limbo foliar desplegado. 
determinar si una semilla es viable o no. Estas últimas al apretarlas se sentían vacías o se las observaba en proceso de descomposición.

El diseño experimental fue completamente al azar con un arreglo factorial $4 \times 2$ (momento de siembra $\mathrm{x}$ tipo de almacenamiento) con diez repeticiones de diez semillas por tratamiento. Se realizó el análisis de la varianza a los datos para los factores momento de siembra, tipo de almacenamiento y su interacción, de acuerdo al arreglo factorial detallado anteriormente. La comparación de medias entre tratamientos se realizó mediante la prueba de Tukey al $5 \%$ de probabilidad, con la ayuda del software Infostat (Di Rienzo et al., 2019). Las variables porcentaje de germinación $(\% \mathrm{G})$ y supervivencia $(\% \mathrm{~S})$ fueron transformadas a la raíz cuadrada para cumplir con los requisitos de normalidad y homogeneidad de varianza.

\section{Estimación Costo operativo/Renta agregada}

El despulpado del fruto provocará cambios en la comercialización de la semilla. En la actualidad, los viveros compran las bolsas de frutos por peso, al retirarle la pulpa habrá mayor cantidad de semillas en bolsas del mismo volumen. Es por ello que se estimó el aumento relativo de número de frutos por bolsa y el incremento de valor que debería asociarse al mismo. Para ello se tomó en cuenta la media del peso de los frutos con y sin pulpa en base al promedio del peso 200 frutos enteros y despulpados.

\section{Clima en la región}

Durante el periodo en que los frutos estuvieron almacenados en el sotobosque ( 26 de mayo al 26 de junio de 2017), las características climáticas para la región tendieron a ser más húmedas con respecto a las normales de la localidad, tanto de precipitaciones como de humedad relativa. Las medias para el periodo de almacenamiento de los frutos fueron de $83,30 \%$ de humedad relativa, 16,1 ${ }^{\circ} \mathrm{C}$ de temperatura y $248 \mathrm{~mm}$ de precipitaciones acumuladas, de acuerdo a datos del Servicio Meteorológico Nacional (in litt, 2018) y Weather Spark (2018).

\section{Resultados}

La observación directa del estado de las semillas de Euterpe edulis post despulpado mecánico no reveló daños en la superficie de estas en ningún caso.

Análisis del poder germinativo de las semillas

A los 140 días de evaluación de cada una de las siembras, no se detectaron diferencias significativas para la interacción entre los factores momento de siembra vs. tipo de almacenamiento, en la variable porcentaje de germinación (Tabla 1, $p>0,05)$. Tampoco hubo diferencias significativas entre los frutos almacenados con y sin pulpa $(84 \pm 2,2 \%$ y $86 \pm 1,8 \%$ de las semillas germinadas respectivamente \pm error estándar, $p>0,05)$, ni para los momentos de siembras $0,1,2$ y $3(82 \pm 3,1 \%$, $85 \pm 3,1 \%, 86 \pm 2,8 \%$ y $87 \pm 2,3 \%$, de las semillas germinadas respectivamente \pm error estándar, $\mathrm{p}>$ $0,05)$. Se destaca el alto porcentaje de germinación de las semillas, superior al $80 \%$, para todos los tratamientos.

En relación a la supervivencia de las semillas y/o plántulas de E. edulis, se contabilizaron los individuos viables (aparente estado latente), muertos y vivos, para cada tratamiento. Para la variable porcentaje de supervivencia no se detectaron diferencias significativas en la interacción entre los dos factores (momento de siembra x tipo de almacenamiento) (Tabla 1, p > 0,05). Tampoco hubo diferencias significativas entre los frutos almacenados con y sin pulpa $(75,8 \pm 2 \%$ y $80,5 \pm 2,3 \%$ de plántulas supervivientes respectivamente \pm error estándar, $\mathrm{p}>0,05)$, ni para los momentos de siembras $0,1,2$ y $3(76 \pm 3 \%, 74,5 \pm 3,4 \%$, $81,5 \pm 2,5 \%$ y $80,5 \pm 3,3 \%$ de plántulas supervivientes respectivamente \pm error estándar, $p>0,05$ ).

Se detectaron diferencias significativas para la interacción entre los factores momento de siembra vs. tipo de almacenamiento en la variable índice de velocidad de germinación (IVG) (Tabla 1, F: $2,37, \mathrm{p}<0,05)$. Los valores mayores de IVG fueron observados para los frutos almacenados despulpados de la siembra $3(0,27 \pm 0,01)$ y los menores para la siembra $0(0,17 \pm 0,01)$.

En la Figura 2 se puede observar la dinámica de la germinación de los frutos almacenados con $\mathrm{y}$ sin pulpa de todas las siembras en conjunto. A los 14 días comenzaron a germinar las semillas de frutos almacenados sin pulpa $(0,25 \%)$, mientras que las semillas de frutos almacenados con pulpa comenzaron a los 28 días (16\%). Por otro lado, las semillas consideradas viables fueron aquellas 


\section{S. Lindner et al. - Aprovechamiento múltiple de E. edulis y conservación del poder germinativo}

Tabla 1. Efecto de la interacción entre momento de siembra y tipo de almacenamiento de los frutos de

$E$. edulis en las variables porcentaje de germinación (G), supervivencia de plántulas (S) e índice de

velocidad de germinación (IVG). Los valores entre paréntesis indican el error estándar. Letras distintas indican diferencias significativas por el test de Tukey $(p<0,05)$.

\begin{tabular}{|ccccc|}
\hline Momento de siembra & $\begin{array}{c}\text { Tipo de } \\
\text { Almacenamiento }\end{array}$ & $\mathbf{G ~ ( \% )}$ & $\mathbf{S}(\%)$ & IVG \\
\hline 0 & ACP & $84(5) \mathrm{A}$ & $77(3) \mathrm{A}$ & $0,18(0,01) \mathrm{AB}$ \\
0 & ASP & $80(3,9) \mathrm{A}$ & $75(5,4) \mathrm{A}$ & $0,17(0,01) \mathrm{A}$ \\
1 & ACP & $86(5) \mathrm{A}$ & $70(4,5) \mathrm{A}$ & $0,18(0,01) \mathrm{AB}$ \\
1 & ASP & $84(4) \mathrm{A}$ & $79(4,8) \mathrm{A}$ & $0,22(0,01) \mathrm{BC}$ \\
2 & ACP & $84(4) \mathrm{A}$ & $79(3,5) \mathrm{A}$ & $0,20(0,01) \mathrm{ABC}$ \\
2 & ASP & $88(3,9) \mathrm{A}$ & $84(3,7) \mathrm{A}$ & $0,24(0,02) \mathrm{CD}$ \\
3 & ACP & $82(3,9) \mathrm{A}$ & $77(5) \mathrm{A}$ & $0,23(0,01) \mathrm{CD}$ \\
3 & ASP & $92(1,3) \mathrm{A}$ & $84(4,3) \mathrm{A}$ & $0,27(0,01) \mathrm{D}$ \\
\hline
\end{tabular}

Abreviaturas: ACP, Almacenamiento con pulpa; ASP, Almacenamiento sin pulpa.

que presentaron un estado latente, es decir, en condiciones adecuadas podrían germinar, pues no estuvieron ni podridas, ni vacías. Si bien este valor fue mayor en los frutos fueron almacenados con la pulpa, la diferencia no fue significativa $(\mathrm{p}>0,05)$.

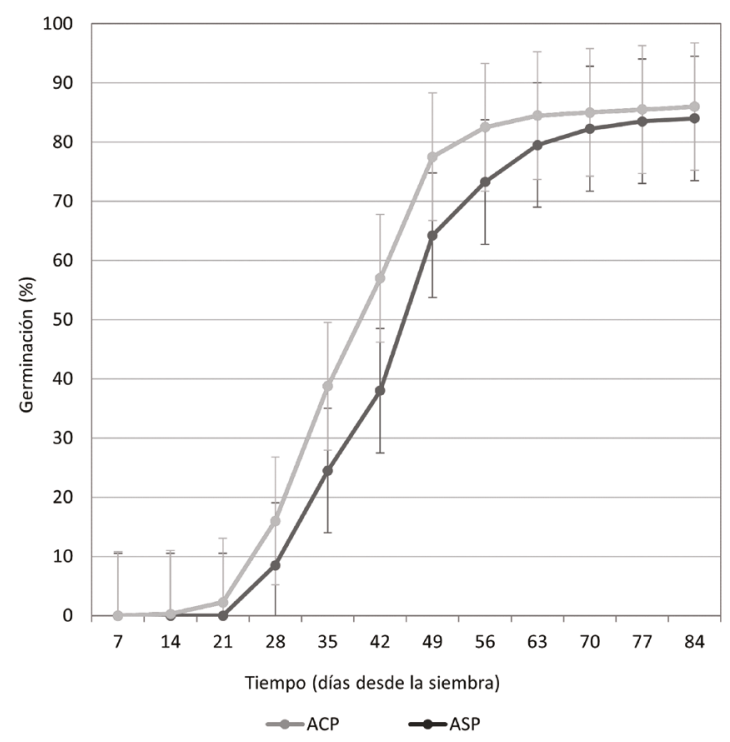

Fig. 2. Germinación acumulada (\%) en el tiempo (días desde la siembra) \pm error estándar de las semillas de $E$. edulis según la condición de almacenamiento de los frutos. Abreviaturas: ACP, Almacenamiento con pulpa; ASP, Almacenamiento sin pulpa.
Tampoco hubo diferencias significativas $(p>0,05)$ para la variable mortalidad de semillas/plántulas entre los frutos almacenados enteros o despulpados.

\section{Estadios de crecimiento}

La Figura 3 compara el promedio observado en cada estadio de crecimiento en las cuatro siembras para las semillas de ambas condiciones de almacenamiento. Para frutos almacenados sin pulpa (Fig. 3A) se observa que en promedio el desarrollo ocurrido a partir de cada semilla fue más rápido en la última siembra (día 30 de almacenamiento) y menor en la primera siembra (día 1 de almacenamiento). Con las semillas provenientes de frutos almacenados con pulpa, en principio ocurre lo mismo, pero a partir de la semana 14 no se observa una conducta clara en ninguna de las siembras, los valores promedio de velocidad para alcanzar los distintos estadios en cada siembra se superponen (Fig. 3B).

Estimación del incremento del valor comercial de las semillas despulpadas

En nuestra región de estudio el promedio del peso fresco por fruto, en base a 200, fue de 1,45 gramos por unidad (desvío estándar: $0,25 \mathrm{~g}$ ). Se extrajeron en promedio $0,47 \mathrm{~g}$ de pulpa/fruto (desvío estándar: $0,20 \mathrm{~g}$ ) lo que representa que en promedio un 33,2 \% del peso del fruto, es pulpa. Por lo tanto, se puede estimar que en una bolsa de $50 \mathrm{~kg}$ de semillas despulpadas y considerando 

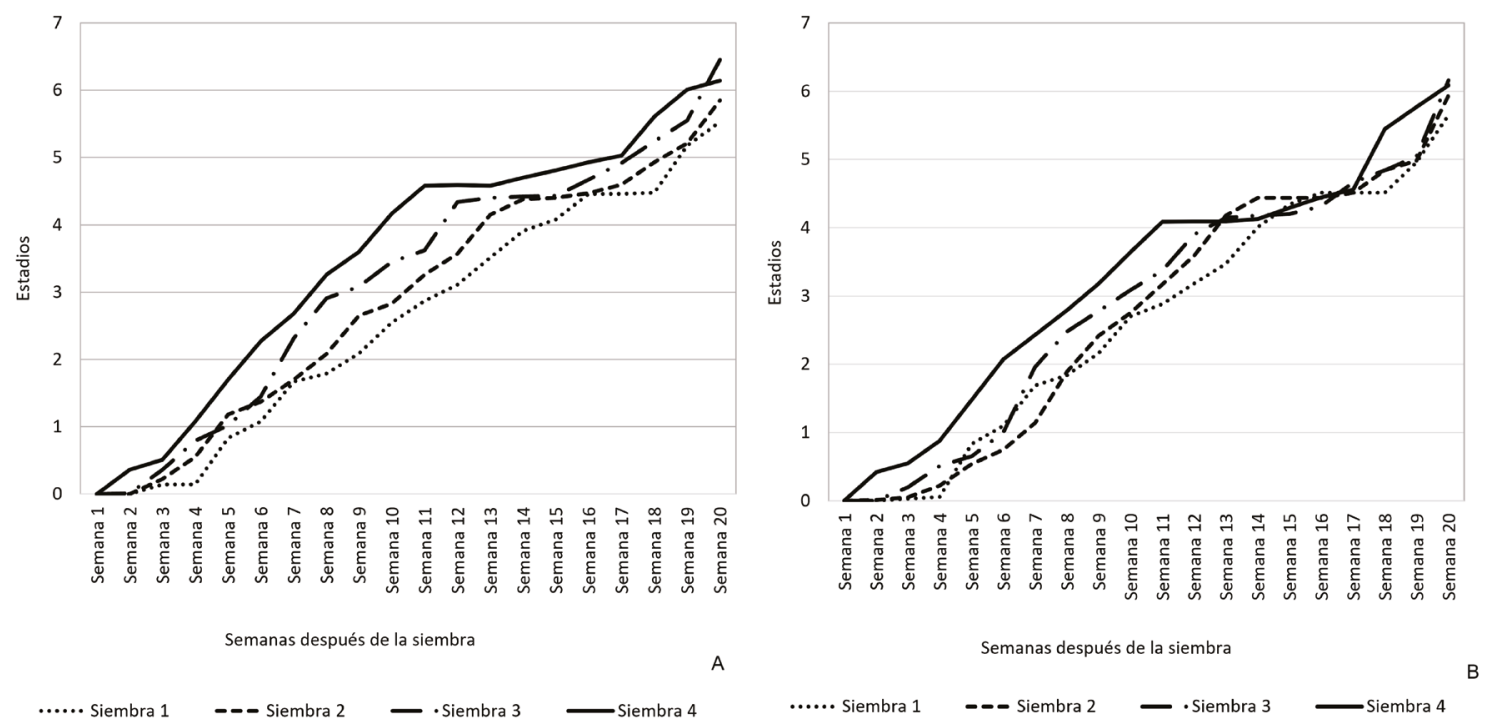

Fig. 3. Progreso de los estadios de desarrollo de las plántulas de $E$. edulis para todas las siembras y condiciones de almacenamiento de los frutos. Referencias: A: Frutos almacenados sin pulpa. B: Frutos almacenados con pulpa.

que a estas se las almacena con el hollejo para aportar humedad, hay aproximadamente un $30 \%$ más de semillas. Entonces sugerimos que el precio de venta del kilogramo de semillas aumente en un $30 \%$ por el aumento en la cantidad de semillas contenidas y en un $20 \%$ más por ser semillas seleccionadas (despulpadas, con mayor velocidad de germinación, en óptimo estado de maduración y con menos residuos vegetales como ramitas, hojarascas, etc., estimación cualitativa). Es decir, en total el aumento será de un $50 \%$ en el valor de las semillas al momento de comercializarlas.

\section{Discusión}

La condición de almacenamiento de los frutos y el tipo de despulpado no afectan la germinación de las semillas de Euterpe edulis. Estos resultados concuerdan con lo obtenido por Cursi \& Cicero (2014) que encontraron que el desempeño fisiológico de las semillas de E. edulis no se ve afectado por el tipo de despulpado (manual o mecánico). En cambio, Martins et al. (2004) encontraron que las semillas de E. edulis almacenadas sin pulpa presentan una mayor germinación y vigor al comparar con frutos almacenados enteros.
Los ensayos realizados hasta la fecha en relación a la germinación de semillas de palmito (E. edulis) proceden de Brasil. La mayor parte de los mismos evalúan el impacto del despulpado mecánico en la viabilidad de las semillas, sin tener en cuenta el almacenamiento como variable de manejo. En ese contexto, se hallaron resultados favorables en cuanto a la germinación de las semillas post despulpadas (Cursi \& Cicero, 2014; Ball \& Brancalion, 2016; Schulz et al., 2016). Asimismo, en frutos despulpados mecánicamente se registraron valores de germinación mayores al $90 \%$ de las semillas, lo que sugiere que el tratamiento podría generar un tipo de escarificación que favorece la germinación (Bovi \& Cardoso, 1976; Bovi, 1990; Martins et al., 2004; Mac Fadden, 2005; Pupo, 2007; Aguiar et al., 2017; Guimarães et al., 2018). De acuerdo a trabajos realizados respecto a los usos del fruto de la palmera E. edulis, se ha observado que la despulpadora al golpear los frutos con las aspas, no solo retira la pulpa (ectocarpo), sino que también remueve parcialmente las fibras mesocárpicas, lo que altera y ablanda su cubierta; en consecuencia, penetran mejor el agua y los gases y se acelera el rompimiento de la dormancia de la misma (William, 1991). Lo que podría explicar el efecto "sincronizador" observado en la germinación 


\section{S. Lindner et al. - Aprovechamiento múltiple de E. edulis y conservación del poder germinativo}

de las semillas almacenadas provenientes de frutos despulpados de forma mecánica en nuestro ensayo.

Bovi (1990) y Aguiar et al. (2017) concluyen que la pulpa oleaginosa de este fruto y la presencia de un opérculo ceroso, contribuyen a disminuir el porcentaje de germinación, por ser aquella un sitio propicio para el desenvolvimiento de microorganismos y el opérculo impedir la penetración del agua. En nuestro ensayo sería necesario evaluar si el almacenamiento junto con el hollejo -dejado para promover el mantenimiento de humedad- constituye un medio propicio para el desarrollo de microorganismos que afecten a las semillas.

Con relación a la germinación natural de los frutos de E. edulis sin ningún tratamiento pre germinativo, Reis \& Kageyama (2000) observaron que el $45 \%$ de las semillas que permanecen después de 150 días en el manto (suelo de materia orgánica muerta) mantienen su capacidad de germinación. Carvalho (1994) estimó que el proceso germinativo medio ocurre entre los $30 \mathrm{y}$ 170 días posteriores a la siembra. Cursi \& Cicero (2014) hallaron que la emergencia se estabiliza a los 135 días, Guimarães et al. (2018) en cambio observó que la emergencia de las plántulas de frutos sin y con pericarpio, comenzó a los 30 y 44 días después de la siembra. En nuestro estudio, una alta proporción de los frutos almacenados sin pulpa germinó entre los 40 a 45 días. Lo que sugiere que el tratamiento mecánico ofició de escarificador y que probablemente la selección de frutos con el mismo estado madurativo contribuyó a la homogeneización de las semillas.

En cuanto al análisis del índice de velocidad de germinación (IVG), para la especie existen antecedentes de IVG de 0,281 para semillas provenientes de frutos sin el pericarpio y 0,146 con el pericarpio (Guimarães et al. 2018), mientras que Tavares et al. (2008) encontraron valores que variaron entre 0,115 y 0,154 para semillas sembradas en diferentes condiciones de sombra. Por lo tanto, nuestros resultados de IVG $(0,27$ para el mejor tratamiento donde los frutos fueron almacenados sin pulpa y el momento de siembra número tres) son similares a los reportados previamente en la bibliografía.

Finalmente, en la comercialización, debería considerarse un aumento al precio de los frutos despulpados, ya que es necesario, previo al despulpado mecánico, realizar una selección de los frutos para descartar aquellos verdes, duros o secos. Además de que las semillas provenientes de frutos despulpados germinarán más rápido y de forma sincronizada. Será necesario poner a prueba y consensuar con los compradores de los frutos de "palmito".

\section{Conclusiones}

El efecto del almacenamiento de los frutos despulpados en la germinación de las semillas de Euterpe edulis Mart. no es una limitante para la incorporación de la extracción de la pulpa previa comercialización para la producción de plantines.

La posibilidad del doble aprovechamiento de los frutos (pulpa y semillas) favorecerá no solo a las economías regionales, sino también permitirá aumentar la valorización de los palmitales en las chacras, promoviendo la conservación por el uso de los remanentes del bosque local.

Se requieren nuevos estudios para definir condiciones más adecuadas para el almacenamiento de los frutos y que contemplen extender este periodo, además de profundizar en el proceso de despulpado y canales de comercialización, buscando preservar las cualidades de la pulpa de los frutos.

Los resultados generan una base técnica con recomendaciones de manejo de los frutos destinados al aprovechamiento múltiple y para la readecuación del valor comercial de los frutos despulpados.

\section{Contribuciones de los Autores}

SL realizó la investigación, estableció y monitoreó los ensayos; analizó los resultados, diseño las figuras y redactó el documento original. PT revisó y amplió los análisis, modificó el texto original, amplió la revisión bibliográfica y la discusión y adaptó las figuras al formato actual. DSG y NIH conceptualizaron el estudio, definieron la metodología y asistieron en las tareas de campo. NIH dirigió el proyecto, obtuvo y administró el financiamiento. Y participó en todas las etapas de redacción del trabajo. Todos los autores revisaron y aprobaron la última versión del manuscrito. 


\section{Agradecimientos}

A los integrantes de la familia Karadya, Luciana y Julián, y a sus vecinos (María, Antonio y sus familias), a la Reserva Yvytú y San Sebastián, por el apoyo y ayuda durante las tareas de campo. A las familias de Península de Andresito y a María, la maestra de la escuela $N^{\circ} 891$, por abrir sus puertas tan amablemente. A Maru Iezzi y Virginia Diaz Villa por su orientación en los análisis estadísticos. $\mathrm{Al}$ editor de este artículo Federico Mollard, por sus minuciosas revisiones y sugerencias. Este trabajo es la tesis de grado de SL. Se contó con becas de postgrado de CONICET para DSG y PT y financiamiento parcial de diferentes proyectos: PNUD ARG 15/G 53, UNaM 16/F1088-PDTS, UNaM 16/F084 y PICT-2015-1578.

\section{Bibliografía}

AGUIAR, F. F. A., S. KANASHIRO, P. GIAMPAOLI, V. A. MODOLO, J. D. AGUIAR \& A. R. TAVARES. 2017. Effects of light, temperature and mesocarp on seed germination of Euterpe edulis (Juçara-Palm). Biosci. J. 33: 881-885. https://doi.org/10.14393/BJ-v33n4a2017-36736

BALL, A. A. \& P. H. S. BRANCALION. 2016. Governance challenges for commercial exploitation of a non-timber forest product by marginalized rural communities. Environm. Conserv. 43: 208220.

BARROSO, R. M., A. REIS \& N. HANAZAKI. 2010. Etnoecologia e etnobotânica da palmeira juçara (Euterpe edulis Martius) em comunidades quilombolas do Vale do Ribeira, São Paulo. Acta Bot. Bras. 24: 518-528. https://doi.org/10.1590/S0102-33062010000200022

BOVI, M. L. A. 1990. Pré-embebição em água e porcentagem e velocidade de emergência de sementes de Palmiteiro. Bragantia 49: 11-22. http://dx.doi.org/10.1590/S0006-87051990000100002

BOVI, M. L. A. \& M. CARDOSO. 1976. Germinação de sementes de palmiteiro (Euterpe edulis Mart.). Bragantia 34: 29-34. http://dx.doi.org/10.1590/S0100-6762200600050000

CARVALHO, P. E. R. 1994. Espécies florestais brasileiras: recomendações silviculturais, potencialidades e uso da madeira. Vol. 1. Colombo: EMBRAPA-CNPF.
CHEDIACK, S. E. 2008. Aprovechamiento sustentable del Palmito Misionero. En: ACEÑOLAZA, F. (ed.), Temas de la biodiversidad del litoral III. Miscelánea 17: 309-316.

CLEMENT, C. R., E. LLERAS \& J. VAN LEEUWEN. 2005. O potencial das palmeiras tropicais no Brasil: acertos e fracassos das últimas décadas. Agrociencia 9: 67-71.

CURSI, P. R. \& S. M. CICERO. 2014. Fruit processing and the physiological quality of Euterpe edulis Martius seeds. J. Seed Sci. 36: 134-142. https://doi.org/10.1590/2317-1545v32n2847

DE BRITO, E. S., M. C. P. DE ARAUJO, R. E. ALVES, C. CARKEET, B. A. CLEVIDENCE \& J. A. NOVOTNY. 2007. Anthocyanins present in selected tropical fruits: acerola, jambolão, jussara, and guajiru. J. Agric. Food Chem. 55: 9389-9394. https://doi.org/10.1021/jf0715020

DI RIENZO, J. A., F. CASANOVES, M. G. BALZARINI, L. GONZÁLEZ, M. TABLADA \& C. W. ROBLEDO. 2019. Centro de Transferencia InFoStat, FCA, Universidad Nacional de Córdoba, Argentina. Disponible en: http://www. infostat.com.ar.

FELZENSZWALB, I., M. R. DA COSTA MARQUES, J. L. MAZZEI \& C. A. AIUB. 2013. Toxicological evaluation of Euterpe edulis: a potential superfruit to be considered. Food Chem. Toxicol. 58: 536-544.

FURLAN, V., L. CARIOLA, D. GARCÍA \& N. I. HILGERT. 2015. Caracterización de los sistemas agroforestales familiares y estrategias de uso del ambiente en el Bosque Atlántico Argentino. Gaia Scientia 9: 69-81.

GARCIA, D. S., N. I. HILGERT \& M. S. REIS. 2020. La Palmera Euterpe edulis Mart., una especie clave para la conservación de los remanentes de Bosque Atlántico en Argentina. En: HILGERT, N. I., M. L. POCHETTINO \& J. E. H. BERMEJO (eds.), Palmeras NUS al Sur de la América Austral, pp. 58-81. CultIVA, Programa Iberoamericano de Ciencia y Tecnología para el Desarrollo, CYTED.

GÓMEZ RESTREPO, M. L. 2004. Estimación de la capacidad germinativa y el vigor de las semillas de diomate (Astronium graveolens Jacq.) sometidas a diferentes tratamientos y condiciones de almacenamiento. Revista Fac. Nac. Agron. Medellín 57: 2218-2232

GUIMARÃES, L. A. D. O. P., R. G. DE SOUZA, M. L. DAN, \& M. A. D. GUIMARÃES. 2018. Emergence and vigor of Euterpe edulis seedlings under shading 


\section{S. Lindner et al. - Aprovechamiento múltiple de E. edulis y conservación del poder germinativo}

levels and the presence and absence of the pericarp. Idesia (Arica) 36: 49-56. http://dx.doi.org/10.4067/S0718-34292018000100049.

HENDERSON, A. 2000. The Genus Euterpe in Brazil. In: REIS M. S. \& A. REIS (eds.), Euterpe edulis Martius - (Palmitero) Biologia, Conservação e Manejo, pp. 01-22. Herbário Barbosa Rodrigues, Itajai.

HILGERT, N. I., D. S. GARCÍA, P. THALMAYR, S. LINDNER \& M. S. REIS. 2019. El Jejy'a (pulpa de frutos de Euterpe edulis Mart.). El Súper Alimento que Contribuye a la Conservación y al Desarrollo Familiar en el Bosque Atlántico de Misiones. Congreso Argentino de Agroecología. Mendoza.

KANG, J., Z. LI, T. WU, G. S. JENSEN, A. G. SCHAUSS \& X. WU. 2010. Anti-oxidant capacities of flavonoid compounds isolated from acai pulp (Euterpe oleracea Mart.). Food Chem. 122: 610-617. https://doi.org/10.1016/j.foodchem.2010.03.020

KELLER, H. A. 2009. Plantas textiles de los guaraníes de Misiones, Argentina. Bonplandia 18: 29-37.

MAC FADDEN, M. J. 2005. A Produção de açaí a partir do processamento dos frutos do palmiteiro (Euterpe edulis Martius) na Mata Atlântica. Disertación de Maestría. Universidad Federal de Santa Catarina, Brasil.

MAGUIRE, J. D. 1962. Speed of germination - Aid in selection and evaluation for seedling emergence and vigor 1. Crop Sci. 2: 176-177.

MARTINS, C. C., M. L. A. BOVI, J. NAKAGAWA \& G. GODOY JÚNIOR. 2004. Temporary storage of jussara palm seeds: effects of time, temperature and pulp on germination and vigor. Hort. Brasil. 22: 271-276.

https://doi.org/10.1590/S0102-05362004000200021

MILANESI, L. S., N. PERONI \& M. S. REIS. 2013. Use of the palm Euterpe edulis Martius in landscape units managed by migrants of German origin in Southern Brazil. J. Ethnobiol. Ethnomed. 9: 1-11. https://doi. org/10.1186/1746-4269-9-47

MYERS, N., R. A. MITTERMEIER, C. G. MITTERMEIER, G. A DA FONSECA \& KENT, J. 2000. Biodiversity hotspots for conservation priorities. Nature 403: 853-858. https://doi.org/10.1038/35002501

POULOSE, S. M., D. R. FISHER, J. LARSON, D. F. BIELINSKI, A. M. RIMANDO, A. N. CAREY, A. G. SCHAUSS \& B. SHUKITT-HALE. 2012. Anthocyanin-rich açai (Euterpe oleracea Mart.) fruit pulp fractions attenuate inflammatory stress signaling in mouse brain BV-2 microglial cells. $J$. Agric. Food Chem. 60: 1084-1093.

https://doi.org/10.1021/jf203989k

PUPO, P. S. S. 2007. Manejo de frutos de palmeira juçara (Euterpe edulis M.) para a obtenção de polpa e sementes como produtos florestais não madeireiros (PFNM) em Mata Atlantica. Disertación de Maestría. Universidad Estadual de Campinas, Brasil.

QUEIROZ, M. H. 2000. Biologia do Fruto, da Semente e da Germinação do Palmiteiro Euterpe edulis. In: REIS M. S. \& A. REIS (eds.), Euterpe edulis Martius - (Palmitero) Biologia, Conservação e Manejo, pp. 39-59. Herbário Barbosa Rodrigues, Itajai.

REIS, A. 1995. Dispersión de semillas de Euterpe edulis Martius (Palmae) en una floresta umbrofila densa montaña de la costa atlántica en Blumenau, Tesis Doctoral. Universidad Estadual de Campinas, Brasil.

REIS, A., M. T. S. PAULILO, E. M. NAKAZONO \& S. VENTURI. 1999. Efeito de diferentes níveis de dessecamento na germinação de sementes de Euterpe edulis Martius-Arecaceae. Ínsula (Florianópolis). 28: 31-42

REIS, M. S., A. C. FANTINI, R. O. NODARI, A. REIS, M. P. GUERRA \& A. MANTOVANI, 2000. Management and Conservation of Natural Populations in Atlantic Rain Forest: The Case Study of Palm Heart (Euterpe edulis Martius) Biotropica 32: 894-902.

REIS, A. \& P. Y. KAGEYAMA 2000. Dispersão de sementes do Palmiteiro (Euterpe edulis Martius Palmae). In: REIS M.S. \& A. REIS (eds.), Euterpe edulis Martius - (Palmitero) Biologia, Conservação e Manejo, pp. 60-92. Herbário Barbosa Rodrigues, Itajai.

RUFINO, M. S. M., R. E. ALVES, E. S. DE BRITO, J. PÉREZ-JIMÉNEZ, F. SAURA-CALIXTO \& J. MANCINI-FILHO. 2010. Bioactive compounds and antioxidant capacities of 18 non-traditional tropical fruits from Brazil. Food Chem. 121: 996-1002. https://doi.org/10.1016/j.foodchem.2010.01.037

SCHULZ, M., G. D. S. C BORGES, L. V. GONZAGA, A. C. O. COSTA, R. FETT. 2016. Juçara fruit (Euterpe edulis Mart.): Sustainable exploitation of a source of bioactive compounds. Food Res. Int. 89: 4-26. https://doi.org/10.1016/j.foodres.2016.07.027

SERVICIO METEOROLÓGICO NACIONAL. 2018. Disponible en: https://www.smn.gob.ar/. [Acceso: 05/03/2018].

SILVA, F. P., D. A. DE MIRANDA, M. CARNIER, P. K. MAZA, V. T. BOLDARINE, A. S. RISCHITELI, 
F. AVILA, L. P. P. PONTES, A. C. L HACHUL, N. I. P. NETO, E. B. RIBEIROA, C. M. OLLER DO NASCIMENTO, V.V. DE ROSSO \& L. M. OYAMA. 2021. Low dose of Juçara pulp (Euterpe edulis Mart.) minimizes the colon inflammatory milieu promoted by hypercaloric and hyperlipidic diet in mice. J. Funct. Foods 77: 104343. https://doi.org/10.1016/j.jff.2020.104343

TAVARES, A. R., D. P. RAMOS, F. F. A. AGUIAR \& S. KANASHIRO. 2008. Jussara palm seed germination under different shade levels. Hort. Brasil. 26: 492494.

TOMLINSON, P. B. 1990. The structural biology of palms. Oxford University Press, New York.
TREVISAN, A. C. D., A. C FANTINI, A. L. SCHMITTFILHO \& J. FARLEY 2015. Market for Amazonian açaí (Euterpe oleraceae) stimulates pulp production from Atlantic Forest juçara berries (Euterpe edulis). Agroecol. Sustain. Food Syst. 39: 762-781. https://doi.org/10.1080/21683565.2015.1025461

WEATHER SPARK. El clima típico de cualquier lugar del mundo. 2018. Disponible en: https://es.weatherspark. com/m/29574/6/Tiempo-promedio-en-junio-enBernardo-de-Irigoyen-Argentina\#Sections-Rain [Acceso: 05/03/2018].

WILLIAM R. L. 1991. Guía para la manipulación de semillas forestales. FAO, Roma. http://www.fao.org/ docrep/006/AD232S/ad232s00.htm\#TOC 